\title{
Adaptive inventory management system of an industrial enterprise in the unsteady market environment
}

\author{
$N$. Dzenzelyuk ${ }^{1, *}$ \\ ${ }^{1}$ South Ural State University, Chelyabinsk, Russia
}

\begin{abstract}
The article examines the features and problems of methodology of formation of inventory management in a volatile market environment. Currently, there was a need to change the development paradigm of the scaling operations to the more efficient use of resources and capital. In this regard, the high importance of the newly received inventory control problem as an essential component of enterprise resources. The author sets out the basic methodological principles of inventory management systems. The article from the standpoint of the theory of systems and automatic control systems proposed structure adaptive inventory management system. As a criterion of the effectiveness of the joint action is considered the minimum criterion of total costs and expenses associated with the unmet demand. The simulation results confirm the efficiency of the developed model under unforeseen and significant fluctuations in the parameters of stochastic demand.
\end{abstract}

\section{Introduction}

Despite the rather long period of inventory management theory development, the issue of creating effective and practically implemented inventory management systems is still relevant.

The search for new solutions in this area is actively carried out [1-5]. The importance of this area is fairly high due to the high proportion of stocks in the capital of companies as well as low capital productivity of the economy as a whole.

Today's economy has developed an urgent need to move from the paradigm of development through the expansion of operations to the paradigm of the capital productivity and resource efficiency improvement. This is true not only for the Russian economy, but also for the world economy as a whole.

Confirmation of the above is the large number of publications related to the search for a variety of practical solutions in the field of and increasing of assets turnover as a whole and inventory turnover as the most "bound" part of the current assets [6-9].

In this regard, it is sufficient to note that Russian companies have a high current asset to total asset ratio. Amount of inventories is more than 8 percent of economy in Russia, while the return on assets not exceeds 7 percent $[10,11]$.

Thus, the inventories today is one of the most important components that improve profitability and ensure competitiveness of business simply because they continually involve substantial financial resources that form the profits of an enterprise, and efficient use of which requires continuous monitoring. Therefore, the enterprise inventory management means the implementation of inventory control and market environment control, the analysis of their interaction and influence on the operation of the enterprise as the subject of market economy, decisionmaking, aimed at saving money and increasing the rate of assets profit.

\section{Some aspects of construction of model of inventory management system}

The main difficulties of effective current assets management appear, as a rule, in the inventory management system, due to the necessity of sufficiently regular purchase, transportation, storage and sales in an unsteady market with high dynamism and uncertainties relevant to inventory management parameters. This fact makes the focus on the examination of inventory as an unsteady stochastic object of management.

The study of inventory management problems from the proposed positions allowed to formulate the following methodological principles of construction of efficient inventory management systems:

- The principle of the market environment unsteadiness;

- The principle of the management systems adaptivity;

- The principle of adequate complexity and dynamism of management methods and algorithms;

- The principle of the generation of multi-criteria quasi-optimal solutions based on heuristic realtime simulation modelling. 
Without dwelling on the principles (substantively presented by the author in [12]), we note that such basic approach should be used in the construction of models of practically implemented inventory management system (hereinafter, IMS).

Author's development of the model, which meets the formulated principles, is discussed below.

Interpretability, adequacy of the actual conditions and universality are worth noting among the most important requirements for developing of IMS models. Nowadays the development of inventory management theory is distinguished by increasing use of modern analytical and mathematical apparatus of the theory of automatic control systems theory. The most frequently cited are the principle of maximum, dynamic programming, statistical optimization, identifying algorithm and filtering algorithm, adaptive approach, linear and nonlinear programming and invariant strategies class [13-17]. The effective practical implementability in the general case can only be achieved when the derived IMS models simultaneously have all three properties listed above (interpretability, adequacy and universality). However, studies indicate that the actual nature of their interaction does not meet the specified requirements [18].

For this reason, the determination of the morphology and algorithms of simple, adaptive inventory management systems and the justification for their adequacy become the most important problem. It is known that the most rational, and sometimes the only method of study and decisionmaking in the conditions of high uncertainty and complexity of the unsteady object of management is a simulation modelling method [19]. Let us discuss some features of IMS development which meet the above mentioned principles.

The basis of the system is formed by IMS structure proposed by A.A. Pervozvanski [20]. The being studied IMS, in this case, is considered as a management system consisting of an object of management (in our system - stock reserve), and controlling system consisting of a regulator (executive body) - a supplier that operates since the formation of the control signal (in our system - purchase order), which, in turn, is formed considering results of demand forecast as an external disturbance, and measurement of the current level of inventory (the condition of the object of management).

This approach is a focused specification of the classical approach to the management systems construction and can be used for the construction of the morphology of the adaptive system. However, the solutions proposed by A.A. Pervozvanski do not meet the actual conditions of stochasticity and unsteadiness of the economic environment. Moreover A.A. Pervozvanski a priori considered the opportunity to separate the optimal regulation of reserve stock and current inventory, without proving the admissibility of such a separation in a real situation.

Therefore, the universal method of simulation modeling should be used to obtain managerial decisions, in contrast to analytical approaches which provide particular solutions with strong assumptions.

Starting from the fundamentals of applied systems theory and automatic control systems theory and considering the structure proposed by A.A. Pervozvanski, the common morphological structure of IMS on demand can be synthesized (Fig. 1).

Simulation modelling of the proposed structure as an unsteady stochastic system allowed us to estimate the possibility of extension of the results of A.A. Pervozvanski model, to more general and real conditions of the functioning of the IMS as well as to remove a number of fundamentally strong assumptions for known theoretical solutions to the optimal inventory management tasks. When choosing the IMS efficiency criterion, a Du Pont formula was primarily considered for the determination of return on assets, as this formula allows the integral estimation of enterprise efficiency. However, studies by the authors show that, when all types of costs had taken into account, this criterion was reduced to the criterion of aggregate costs minimization, which is used in the subsequent construction and research of the adaptive IMS.

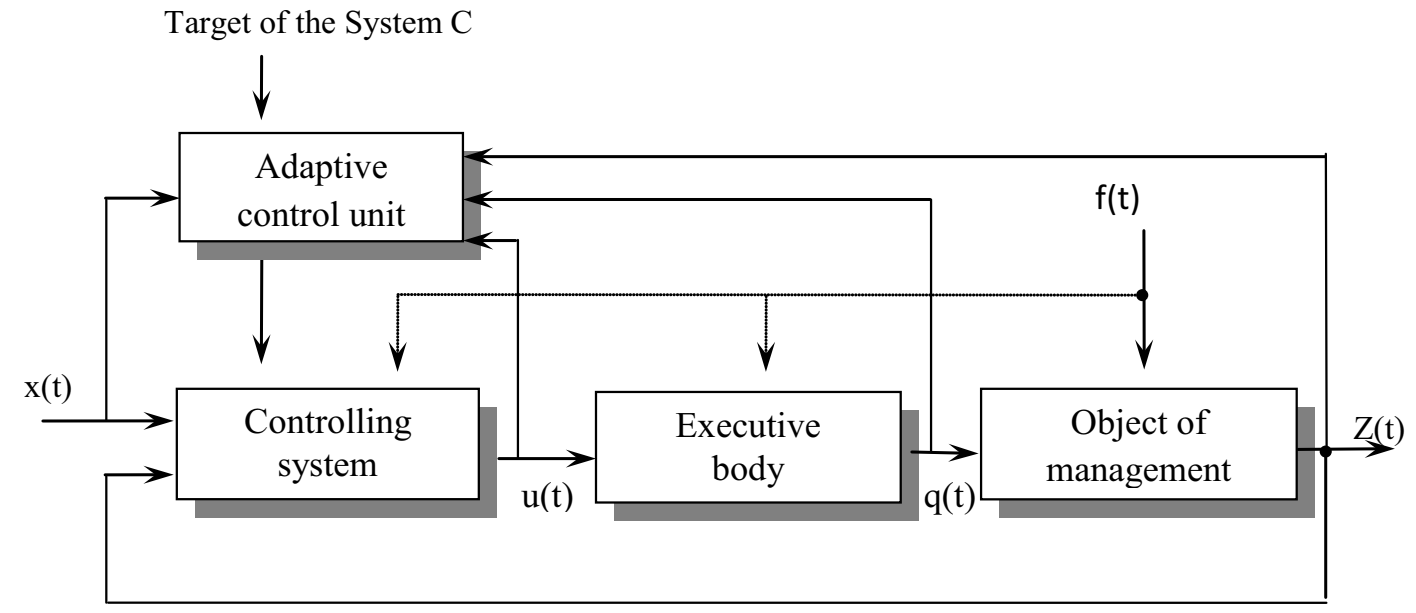

Fig. 1. Common morphological structure of IMS. $x(t)$ - regular environmental disturbance, $u(t)$ - control parameter, $f(t)$ - external environmental action (irregular disturbance), $\mathrm{z}(\mathrm{t})$ - current inventory status, $\mathrm{q}(\mathrm{t})$ - goods delivery. 
Omitting the proof of possibility of substituting unknown parameters of optimal solutions rule by point estimates of such parameters, we should discuss the assumption that environment characteristics are known. Randomized demand $x_{t}$ is chosen to take account of the influence of the external environment (hereinafter the demand and its determinants $-x_{t}, f$ ). The author prefers adaptive approach as attractive and appropriate for two reasons. First, the principle of universality is performed, namely the management system functioning structure and parameters are invariant to changes in the environment. Second, these models offer the simplicity of the structure used. In this case, unlike their original structure, they have the subsystem which allows to estimate of unknown factors. In our case they are $\left(\hat{X}_{t}, \hat{f}\right)$, which recover the correspondence between control parameters $\bar{q}, r$ and scores of factors $\hat{X}_{t}, \hat{f}$, i.e. correction $U(\bar{q}, r)$ by its estimate:

$$
U\left(\bar{q}, r, \bar{x}_{t}, \overline{\bar{f}}\right) \equiv \hat{U}(\bar{q}, r)
$$

Quasi-optimal (adaptive) management strategy, constructed in accordance with the considered method, is a particular case of two-tier $(R, r)$ strategy, symbolically:

$$
U(q, r)= \begin{cases}R-z, & z \leq r, \quad R=q+r \\ 0, & z>r\end{cases}
$$

where $\boldsymbol{z}$-current stock situation;

$\boldsymbol{r}$ - reorder point,

control vector $\boldsymbol{U}(\boldsymbol{q}, \boldsymbol{r})$ is defined by point estimates of mathematical expectation $\bar{x}_{t}$ and variation coefficient $\operatorname{var}\left\{x_{t}\right\}$ of the demand $\left(\overline{\bar{x}}_{t}, \operatorname{var}\left\{x_{t}\right\}\right.$

Then, operative decisive rule for this strategy is to optimize the size $\boldsymbol{q}$ and reorder point $\boldsymbol{r}$ in next period $\boldsymbol{t}+\boldsymbol{T}$ based on procedures of determination and forecasting of the demand $x_{t}$ and estimation $\bar{z}_{t}$ of the current stock situation $z_{t}$, made in basic period $(t)$, so

$$
q_{t+T}=\left\{\begin{array}{ll}
q_{t+T}^{*}, & \widehat{z}_{t} \leq r_{t}+\tau \cdot \overline{\bar{x}}_{t}, \\
0, & \widehat{z}_{t}>r_{t}+\tau \cdot \overline{\bar{x}}
\end{array} \text { when } \boldsymbol{T}>,(3)\right.
$$

where $\tau$-period of delivery,

$$
r_{t+T}^{*}=\left\{r \in \Omega_{r} / C_{r}\left(\operatorname{var}\left\{x_{t}\right\}\right)=\min \right\},
$$

$\Omega_{r}$ - range of possible values of $\boldsymbol{r}$,

$C_{r}\left(\operatorname{var}\left\{x_{t}\right\}\right)$ - aggregate costs of reserve stock management, depending on random component of the demand (variation, presented by indicators estimate (demand variation coefficient $\left.\operatorname{var}\left\{x_{t}\right\}\right)$ ).

Therefore, the derived structure of optimal order quantity $q_{t+T}^{*}$ is similar to optimal order structure, proposed by A.A. Pervozvanski for the linear control law, i.e. consists of deterministic $\bar{q}_{t+T}^{*}$ and random $\tilde{q}_{t+T}^{*}$ components, which are defined separately:

$$
q_{t+T}^{*}=\bar{q}_{t+T}^{*}+\tilde{q}_{t+T}^{*},
$$

The essential difference lies in the procedure of a finding of the components $\bar{q}_{t+T}^{*}$ and $\tilde{q}_{t+T}^{*}$. The finding procedure has significant features of the adaptive method, which fundamentally distinguish the developed strategy of those already known.

First, the definition of deterministic component $\bar{q}_{t+T}^{*}$, which provides accounting of stable (average) component of the demand $\boldsymbol{x}$ by the condition of minimum of storage expenses $\boldsymbol{h}$ and forming $\boldsymbol{A}$ days-onhand inventory (excluding the stockout), although defined by Wilson formula, is based on use of not the target intensity value of the demand $\bar{x}_{t}$, but on its preliminary estimate $\hat{\bar{x}}_{t}$ :

$$
\bar{q}_{t+T}^{*}=\sqrt{\frac{2 \cdot \hat{\bar{x}}_{t} \cdot A}{h}}
$$

Second component of an order $\tilde{q}_{t+T}^{*}$, used for compensation of stochastic component oa the demand $\boldsymbol{x}_{\boldsymbol{t}}$ and necessary to ensure the target level of a satisfied demand, is calculated as the deviation of levels (in next and basic period) of the inventory $\boldsymbol{r}$, which is used as an operation threshold, symbolically:

$$
\tilde{q}_{t+t}^{*}=\Delta \hat{r}_{t}^{*}=\hat{r}_{t}^{*}-\hat{r}_{t-T}^{*}
$$

Thus, this formulation of the synthesis problem in the form of adaptive IMS provides the flexible feedback from the two components of the demand (deterministic and random), so the inventory management capabilities are significantly expanding by including a reserve stock $\boldsymbol{r}$ in a control loop, so the reserve stock becomes a functional relation to the variability of the demand $\boldsymbol{x}_{\boldsymbol{t}}$.

Random order component $\tilde{q}_{t+T}^{*}$ forming block appears similar to operating management channel, where deterministic component $\bar{q}_{t+T}^{*}$ is formed. Obviously, only those parameters of the demand are used which characterize its variation (volatility), such as variation coefficient $\operatorname{var}\left\{x_{t}\right\}$ and standard deviation $\sigma_{X t}$. not a stable component (average intensity $\bar{x}_{t}$ ) is used.

Returning to the criterion of efficiency of considered IMS, the description of its components should be determined.

For example, optimization condition for the parameter $\boldsymbol{r}$ of the reserve stock storage expenses $\boldsymbol{C}_{\boldsymbol{i}}$ and expenses of stockout itself $\boldsymbol{C}_{\boldsymbol{v} \boldsymbol{\eta}}$ is written as:

$$
r^{*}=\left\{r \in \Omega_{r} /\left(C_{i r}+C_{v \eta}\right)=\min \right\}
$$

where $C_{i r}=k_{i r} r, k_{i r}-$ coefficient that indicates the alternative investment profitability;

$C_{v \eta}=k_{\eta} \cdot \bar{\eta}(r), k_{\eta}-$ costs per day, related to unsatisfied demand because of stockout; 
$\bar{\eta}(r)$ - link function between the average quantity of stockout days $\bar{\eta}$ in considered period and the level of the reserve stock $r$.

A quantity of stockout days $\bar{\eta}$ in any cycle of operating management with duration $T$ by random component of the demand depends on estimate of the demand variability $\operatorname{var}\left\{x_{t}\right\}$, i.e. on particular intensity estimates $\bar{x}_{t}$ and on the standard deviation $\sigma_{X t}$ so as $\bar{\eta}(r) \equiv \bar{\eta}\left(r ; \overline{\bar{x}}_{t} ; \bar{\sigma}_{X t}\right)$. This parameter $(\bar{\eta})$ is also used during the system synthesis for its performance evaluation. As it is interesting enough to control the "loyalty" of the buyer to an "empty" warehouse, this parameter is also used in the system as a controlling action (with estimation of number of stockout days effecting the system parameters).

Of course, in general case of different baselines (laws of demand distribution density and its parameters $)$ a formula for a function $\bar{\eta}\left(r ; \overline{\bar{x}}_{t} ; \bar{\sigma}_{X t}\right)$ is also different, and it can easily be derived experimentally during the simulation modelling as a family of curves $\bar{\eta}\left(r ; \operatorname{var}\left\{x_{t}\right\}\right)$. Latest can be converted into the corresponding family of curves of the costs $C_{v \eta}\left(r ; \operatorname{var}\left\{x_{t}\right\}\right)$ for consideration in the target function $\boldsymbol{C}_{\boldsymbol{r}}$ during definition of the optimum values $\boldsymbol{r}^{*}$ and match making between control parameter $\boldsymbol{r}^{*}$ and demand variation values $\boldsymbol{v a r}\left\{\boldsymbol{x}_{\boldsymbol{t}}\right\}$.

Accordingly, the optimization condition can be written as:

$$
r^{*}=\left\{r \in \Omega_{r} /\left(k_{i r} * r+k_{\eta} * \bar{\eta}\left(r ; \operatorname{var}\left\{x_{t}\right\}\right)\right)=\min \right\}
$$

The structure of adaptive IMS, obtained by the author, reflecting the outlined strategy and relevant to considered inventory management model, is shown in Fig. 2.

The use of such a structure and the proposed strategy can greatly simplify the selection of the optimal control parameters of the IMS.

No doubt, for the estimation of the adaptive solutions effectiveness, i.e. proximity of the adaptive $\hat{U}(q, r)$ and the «optimal» $U^{*}(q, r)$ rules for IMS solutions, it is necessary to evaluate the convergence, consistency and efficiency of estimates of the unknown parameters of demand. It is also important to evaluate the rate of convergence of calculated estimates.

Evaluation of the convergence rate of the demand parameter estimates $\overline{\bar{x}}_{t}, \hat{\sigma}_{X t}$ as well as true values $\bar{x}_{t}$ and $\sigma_{X t}$ in the context of a being solved problem of the IMS construction, is necessary due to limited period of delivery. It is obvious that the formation of the demand parameter estimates should take place within a time not exceeding the period of delivery, taking into account the time of the order $T-\tau$.

Analysis of simulation modeling results indicated that the convergence rate of demand estimates $\overline{\bar{x}}_{t}, \bar{\sigma}_{X t}$ to true values $\bar{x}_{t}, \sigma_{X t}$ with accuracy better than $0,5 \%$ does not exceed the specified values in the range of variation coefficient $\operatorname{var}\left\{x_{t}\right\}$ from 0,2 to 2 , and with $\operatorname{var}\left\{x_{t}\right\}=5$, which is extremely rare in reality, does not exceed the period of delivery.

The research results confirm the constructibility of the considered principles of methodology and inventory management system construction, as well as the performance of the proposed algorithms for the estimation of unknown parameters of demand. Thus, it can be stated that the proposed adaptive strategy and the model of operational inventory management could well be used in harsh environments typical of the unsteady economy, namely in terms of significant and unforeseen fluctuations in the stochastic parameters of demand during delivery period.

\section{Conclusion}

The conducted studies allow to state a constructibility of the proposed methodological principles at the current stage of inventory management theory development and a possibility of construction of an adequate universal adaptive management model with well interpretable results.

The work was supported by Act 211 Government of the Russian Federation, contract № 02.A03.21.0011

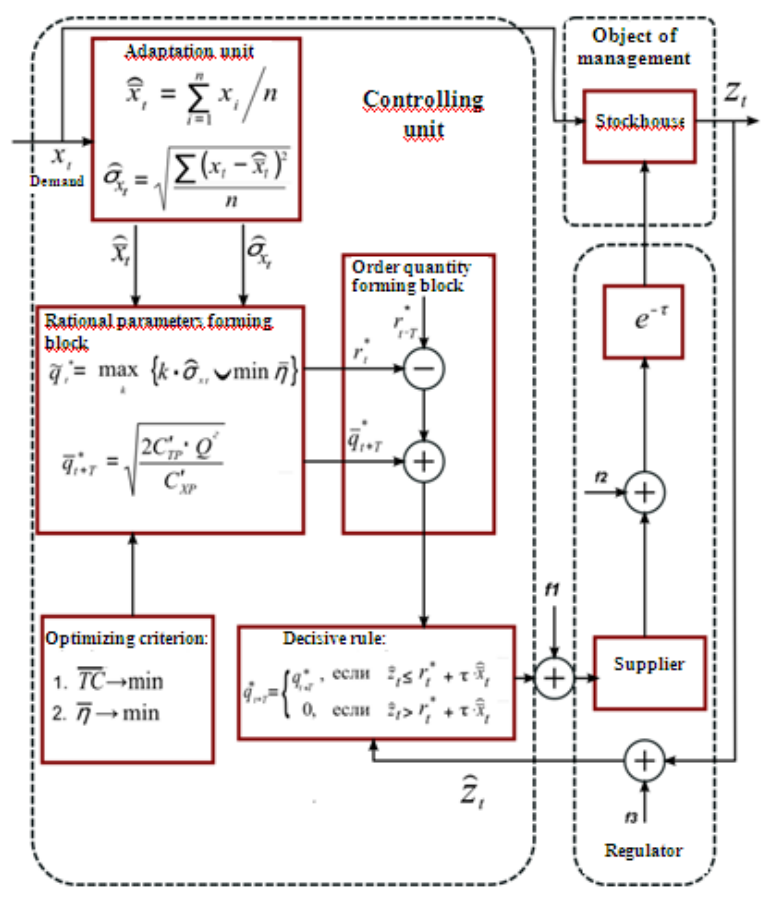

Fig. 2. Adaptive IMS structure.

\section{References}

1. J. Buchan, E. Koenigsberg, Scientific Inventory Management (Prentice-Hall, 1963)

2. A.G. Butrin, Economy of a region, 2, 130-140 (2014) 
3. N.I. Vorobyova, V.S. Lukinskii, V.V. Lukinskii, Logistics and Supply Chain Management, 3(62), (2014)

4. V.S. Lukinskii, Proc. Logistics: modern trends of development: materials of the XIII International scientific-practical Conference, 24 (2014)

5. O.A. Sviridova, Organizational development modeling: the round table reports, Scientific Technologies (2014)

6. I.A. Baev, A.V. Shmidt, Bulletin of Ural Federal University. Series Economics and Management, 3, 50-63 (2012)

7. A.G Butrin, A.I. Kovalyov, Economic analysis: theory and practice, 3 (2011)

8. N.I. Reshetko, Y.I. Bashkatova, Electronic scientific publication of "Naukovedenie", 7, 1 (2015)

9. E.N Troyanova, N.P. Ivantsova, Social sciences, 1, 6-1(9), 103-107 (2015).

10. Russian Finances, 2014: statistics digest (Rosstat, Moscow, 2014)

11. N.S. Dzenzelyuk, Management in Russia and Abroad, 3 (2013)
12. L.A. Baev, N.S. Dzenzelyuk, Y.N. Tarasov, Theoretical and Practical Aspects of Management, 1 (2014)

13. Y.I. Ryzhikov, The theory of lines and inventory management (Piter, Moscow, 2001)

14. E.V. Shikin, A.G. Chkhartishvili, Mathematical methods and models in the management: Educat. Guidance (Delo, Moscow, 2002)

15. V.I. Smagin, S.V.Smagin, Tomsk State University Journal, 290 (2006)

16. V.I. Smagin, S.V.Smagin, Tomsk State University Journal, 3(4) (2008)

17. K.P. Goloskokov, K.A. Davydov, Economic systems Management, 2 (2012)

18. Y.N. Tarasov, N.S. Dzenzelyuk, Economic systems Management: Electronic scientific journal of Kislovodsk institute of economy and law

19. O.I. Babina, Simulation. The Theory and Practice (2009)

20. A.A. Pervozvanski, Mathematical models in production management and inventory management (Nauka, Moscow, 1975) 